\title{
Robust memory responses against influenza vaccination in pemphigus patients previously treated with rituximab
}

\author{
Alice Cho, ${ }^{1,2}$ Bridget Bradley, ${ }^{3}$ Robert Kauffman, ${ }^{1,2}$ Lalita Priyamvada, ${ }^{1,2}$ Yevgeniy Kovalenkov, ${ }^{1,2}$ \\ Ron Feldman, ${ }^{3}$ and Jens Wrammert ${ }^{1,2}$ \\ 'Department of Pediatrics, Division of Infectious Disease, ${ }^{2}$ Emory Vaccine Center, and ${ }^{3}$ Department of Dermatology, Emory \\ University School of Medicine, Atlanta, Georgia, USA.
}

\begin{abstract}
Rituximab is a therapeutic anti-CD20 monoclonal antibody widely used to treat B cell lymphoma and autoimmune diseases, such as rheumatic arthritis, systemic lupus erythematosus, and autoimmune blistering skin diseases (AIBD). While rituximab fully depletes peripheral blood B cells, it remains unclear whether some preexisting $B$ cell memory to pathogens or vaccines may survive depletion, especially in lymphoid tissues, and if these memory B cells can undergo homeostatic expansion during recovery from depletion. The limited data available on vaccine efficacy in this setting have been derived from rituximab-treated patients receiving concomitant chemotherapy or other potent immunosuppressants. Here, we present an in-depth analysis of seasonal influenza vaccine responses in AIBD patients previously treated with rituximab, who generally did not receive additional therapeutic interventions. We found that, despite a lack of influenza-specific memory B cells in the blood, patients mount robust recall responses to vaccination, comparable to healthy controls, both at a cellular and a serological level. Repertoire analyses of plasmablast responses suggest that they likely derive from a diverse pool of tissue-resident memory cells, refractory to depletion. Overall, these data have important implications for establishing an effective vaccine schedule for AIBD patients and the clinical care of rituximab-treated patients in general and contribute to our basic understanding of maintenance of normal and pathogenic human B cell memory.
\end{abstract}

\begin{abstract}
Authorship note: R. Feldman and J. Wrammert contributed equally to this study.
\end{abstract}

Conflict of interest: The authors have declared that no conflict of interest exists.

Submitted: February 9, 2017 Accepted: May 16, 2017 Published: June 15, 2017

\section{Reference information:} JCI Insight. 2017;2(12):e93222. https://doi.org/10.1172/jci. insight. 93222

\section{Introduction}

Influenza infection is a leading cause of morbidity and mortality worldwide $(1,2)$, especially in young children, the elderly, and immune-compromised individuals (3). Influenza vaccination is often recommended to these target populations as an effective preventative measure, although vaccine responses in these patient populations is relatively poor compared with healthy vaccinees $(3,4)$. Since serum antibodies are a correlate of protection for vaccine efficacy in humans (5), it is clinically important to study vaccine responses in patients who are receiving medication, or who have a disease, that modulates B cell responses.

Rituximab is a B cell-depleting anti-CD20 monoclonal antibody developed to treat B cell lymphoma (6). It is FDA approved to treat rheumatoid arthritis (RA) and antineutrophil cytoplasmic autoantibody vasculitis (7). There is increasing off-label use for B cell-mediated autoimmune disorders, such as systemic lupus erythematosus and autoimmune blistering skin diseases (AIBD) (8,9). Rituximab targets all B cells, except early precursor pro-B cells and long-lived plasma cells that do not express CD20 $(10,11)$. Thus, it has little effect on preexisting serum antibody titers produced by long-lived plasma cells, such as antibodies against childhood vaccines, including tetanus or meningitis $(6,10)$, but completely eliminates peripheral B cell memory. Depletion of peripheral B cells typically lasts for 6-9 months, with numbers recovering after 1 year (12). Despite recovery of total B cell numbers, the repopulated B cell compartment consists largely of naive cells, and total memory B cells (MBCs) remain almost absent in peripheral blood, even 5 years after treatment (13). It is unknown whether the very small number of MBCs that are detected represent cells that escaped depletion or are newly generated memory cells. It is also unclear if antigen-specific MBCs can avoid depletion in lymphoid organs (14) and/or possibly expand through homeostatic mechanisms, as is the case for T cells (15). 
Table 1. Characteristics of subjects at the time of enrollment

\begin{tabular}{|c|c|c|}
\hline & Patients $(n=23)$ & Healthy controls $(n=28)$ \\
\hline $\operatorname{Sex}(M / F)$ & $8 / 15$ & $9 / 19$ \\
\hline Age in years, median (range) & $51(28-73)$ & $45(22-70)$ \\
\hline \multicolumn{3}{|l|}{ Ethnicity } \\
\hline European descent & 14 & 15 \\
\hline African American & 7 & 12 \\
\hline Other & 2 & 1 \\
\hline Received influenza vaccination during 2011-2013 & $16^{A}$ & 20 \\
\hline \multicolumn{3}{|l|}{ Enrollment } \\
\hline 2014/15 flu season & 13 & 14 \\
\hline 2015/16 flu season & 10 & 14 \\
\hline Time since rituximab dose in months, median (range) & $11(5-24)$ & \\
\hline 5-11 months after rituximab (early) & 13 & \\
\hline At least 12 months after rituximab (late) & 10 & \\
\hline \multicolumn{3}{|l|}{ No. of previous rituximab cycles } \\
\hline 0 & 12 & \\
\hline 1 & 7 & \\
\hline 2 & 2 & \\
\hline 4 & 2 & \\
\hline \multicolumn{3}{|l|}{ Other medications at time of vaccination } \\
\hline None & 16 & \\
\hline Cellcept & 3 & \\
\hline Azathioprine & 2 & \\
\hline Prednisone & 1 & \\
\hline Prednisone + Cellcept & 1 & \\
\hline \multicolumn{3}{|l|}{ Disease type } \\
\hline Pemphigus vulgaris & 14 & \\
\hline Pemphigus foliaceus & 5 & \\
\hline Mucous membrane pemphigoid & 3 & \\
\hline Bullous pemphigoid & 1 & \\
\hline
\end{tabular}

${ }^{A}$ Four patients were unable to recall vaccination history. $M$, male; $F$, female.
AIBD are a group of potentially fatal B cell-mediated autoimmune disorders affecting skin and/or mucous membranes, including pemphigus and pemphigoid (16-18). Pemphigus results in severe erosions caused by autoantibodies directed against intercellular proteins in the epidermis called desmogleins- 1 and 3 (19, 20). Pemphigoid causes blistering due to autoantibodies specific for proteins in the basement membrane zone between the epidermis and dermis, including collagen XVII $(21,22)$. Historically, AIBD patients have been treated with systemic corticosteroids or adjuvant immunosuppressants (23), but recently, treatment with rituximab has gained popularity (24-29). To our knowledge, there are no reports describing vaccine efficacy in rituximab-treated AIBD patients. Previous studies have reported severely impaired immune responses to influenza vaccination in other types of rituximab-treated patients, primarily RA or lymphoma patients (30-34). A major caveat to these earlier studies is that patients were treated with concomitant immunosuppressive drugs or chemotherapy, making it difficult to determine the effect of the individual drugs on vaccine responses. In contrast, AIBD patients are often weaned off additional immunosuppressive drugs after rituximab depletion, making it possible to study vaccine responses in rituximab-treated patients that are not currently prescribed additional immunosuppressive therapies. It is also possible that underlying autoimmune disease may differentially affect vaccine responses, with AIBD patients reacting differently compared with RA or lymphoma patients. Thus, it is important to study the effect of rituximab on vaccine responses in the context of various diseases.

Here, we measured influenza vaccine responses in AIBD patients that received rituximab therapy 5-24 months earlier and compared them to a matched cohort of healthy control subjects. Surprisingly, we found that, despite the absence of detectable preexisting influenza-specific MBCs in peripheral blood, patients mounted a robust humoral immune response to the vaccine, of comparable magnitude, quality, and repertoire breadth to that observed in healthy vaccinees. Interestingly, these responses had all the hallmarks of a memory recall response. This suggests that substantial numbers of MBCs can survive rituximab depletion, presumably in lymphoid tissues, and act as a source for protective recall responses. These findings have important implications for the clinical care of AIBD patients and our understanding of MBC homeostasis and its role in autoimmune disease etiology as well as vaccine responses in the context of B cell ablative therapies in humans.

\section{Results}

Reconstitution of the B cell compartment in rituximab-treated AIBD patients. We enrolled 23 AIBD patients and 28 healthy controls that received the influenza vaccine during the 2014/15 or 2015/16 influenza season. Recruited patients had received rituximab therapy within the last 24 months (median 11 months), and importantly, at the time of vaccination, the majority were not receiving other immunosuppressive therapies. Healthy controls were demographically matched with those with similar influenza vaccination histories (Table 1). 
A
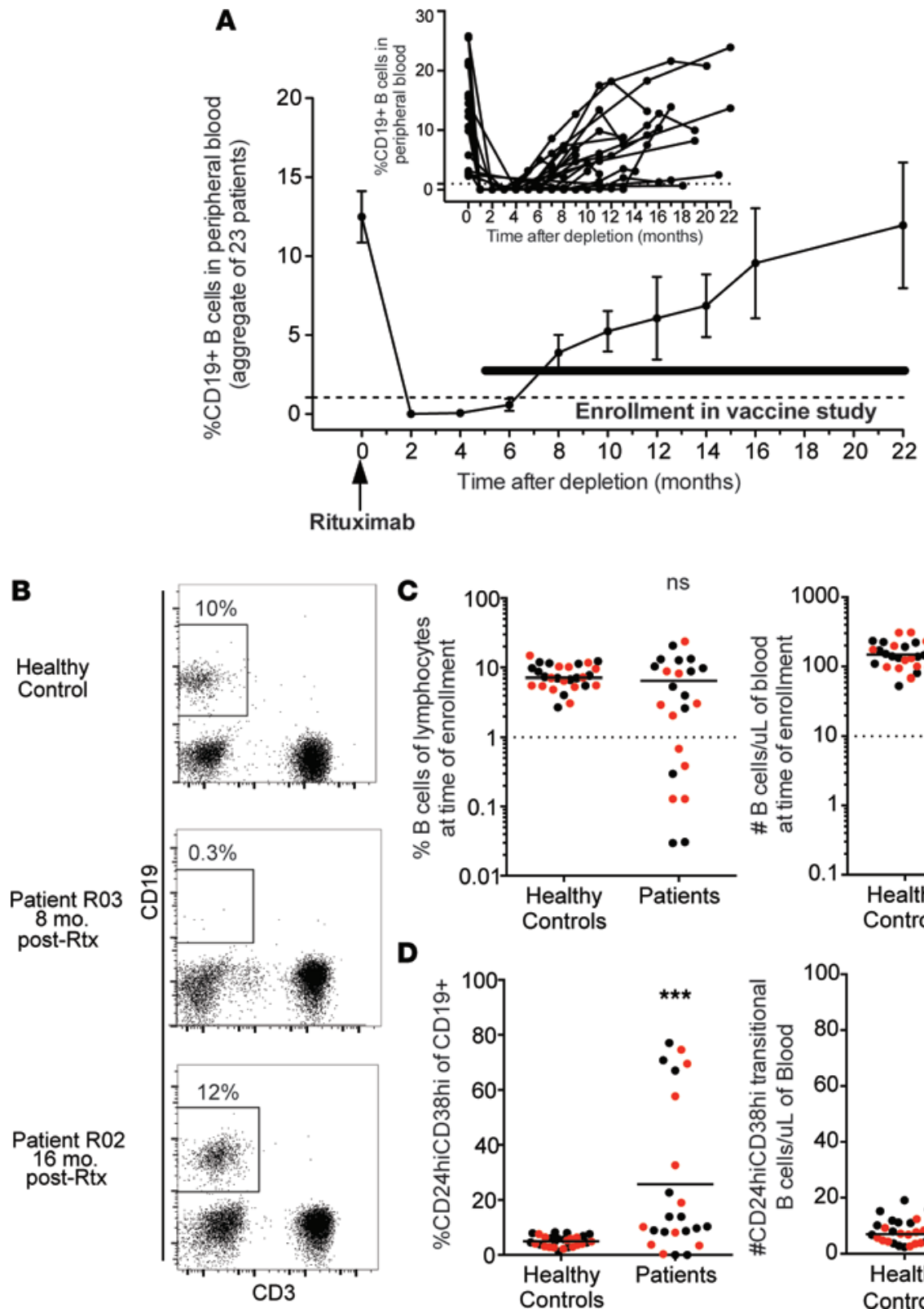

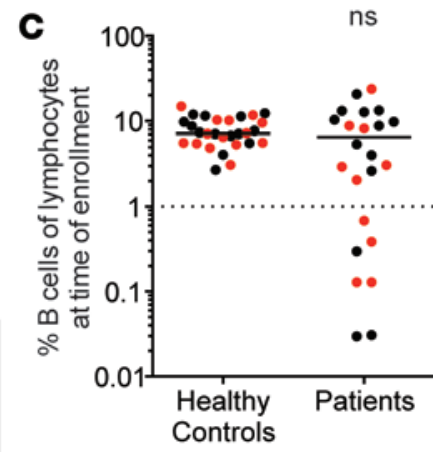

D

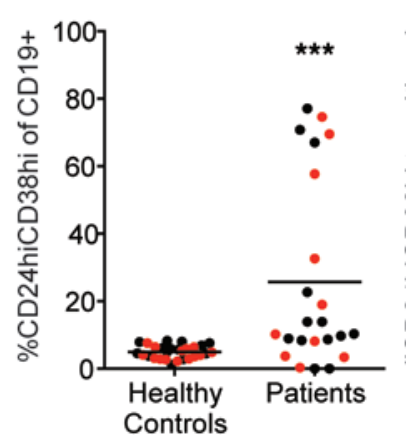

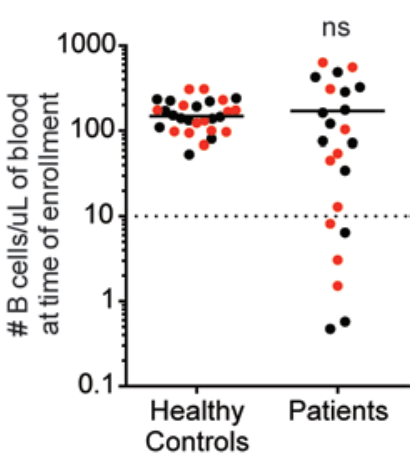

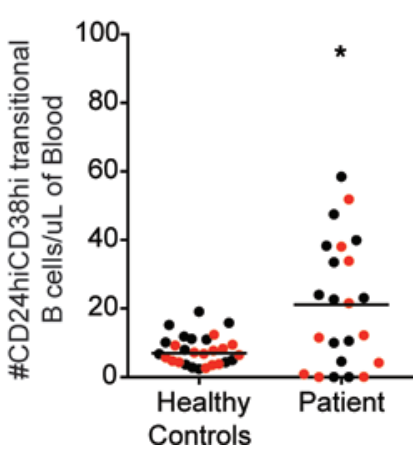

Figure 1. Reconstitution of the B cell compartment after rituximab treatment. (A) Average kinetics (mean \pm SEM) of peripheral blood CD19+ $B$ cells after rituximab therapy. Dotted line represents depletion at $1 \%$ B cells of total lymphocytes. The inset graph depicts B cell depletion kinetics for each individual patient. (B) Representative flow plots of $\mathrm{CD}^{-} \mathrm{CD}^{-} 9^{+}$peripheral $B$ cells, gated on lymphocytes, at time of enrollment. (C) Overall, median frequency and total counts of $B$ cells were comparable between healthy controls and patients. (D) Median frequency and total number of transitional $B$ cells, defined as $\mathrm{CD} 24^{\text {hi }} \mathrm{CD} 38^{\text {hi }}$, were significantly higher in patients than in healthy controls. Mann-Whitney $U$ test was used to analyze data. ${ }^{*} P \leq 0.05$; ${ }^{* * *} P \leq 0.001$. Black circles represent data from the 2014/15 influenza season; red circles represent data from the 2015/16 influenza season.

Numerical reconstitution of the B cell compartment after rituximab treatment typically begins at around 6-9 months, with total B cell numbers returning to a normal range after 1 year $(12,35,36)$ (Figure 1A). During this time, enrolled patients showed improved clinical symptoms and a general decrease in serum autoantibody titers (Supplemental Figure 1; supplemental material available online with this article; https://doi.org/10.1172/jci.insight.93222DS1). We focused our study on patients who were within 5-24 months of receiving rituximab therapy, when patients exhibited disease remission and the B cell compartment was numerically reconstituted, either partially or fully (Figure 1A). Based on pharmacokinetic data for rituximab (12), we expect little rituximab to remain in circulation at this point, which would be important for interpretation. Overall, the frequency of peripheral B cells, defined as CD3-CD19+ lymphocytes (Figure $1 \mathrm{~B})$, did not significantly differ between patients and healthy controls $(4.1 \%$ versus $7.2 \%$, respectively; $P=0.16$ ). Additionally, there was no significant difference when comparing total number of B cells between patients and healthy controls. However, there were 7 patients recruited during early numerical reconstitution with $\mathrm{B}$ cell frequencies of less than $1 \%$ (Figure 1C). It was previously reported that a large wave of $\mathrm{CD} 24^{\mathrm{hi}} \mathrm{CD} 38^{\mathrm{hi}}$ transitional B cells, recently emigrated from the bone marrow, dominate the B cell compartment during initial reconstitution $(36,37)$. Accordingly, the rituximab-treated AIBD patients had 
A

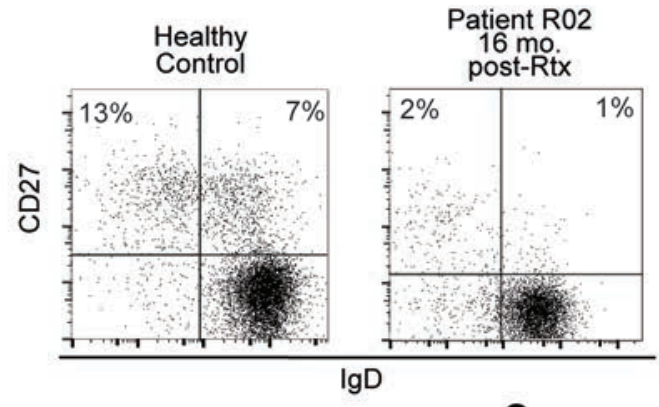

B
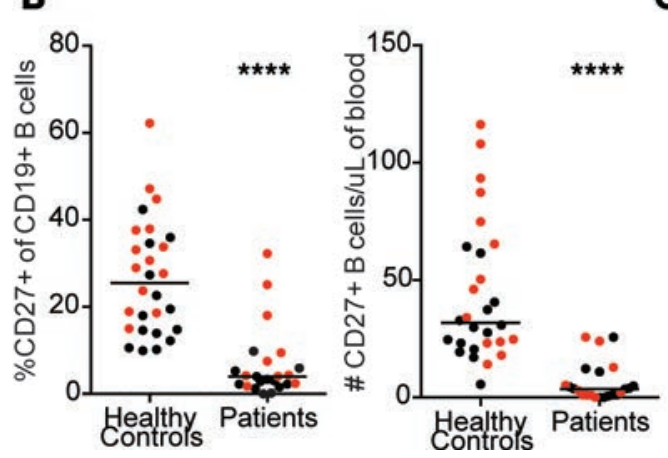

C

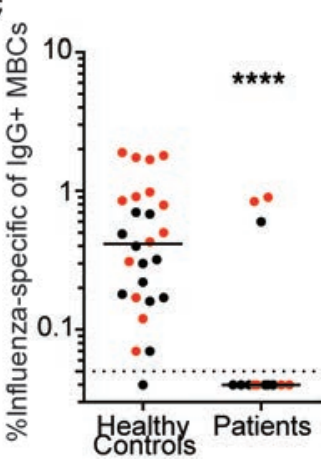

Figure 2. Lack of memory B cells in peripheral blood in rituximab-treated patients. (A) Representative flow cytometry plots showing $\mathrm{CD} 27^{+}$memory B cells (MBCs), gated on CD19+ lymphocytes, at time of enrollment in a healthy control and a patient. (B) The median frequency and total number of MBCs were significantly lower in patients than in healthy controls. (C) Antigen-specific MBCs were stimulated in vitro and detected using an ELISPOT-based assay, as previously described (39, 41). The median frequency of influenza-specific lg $\mathrm{G}^{+} \mathrm{MBC} s$ was significantly lower in patients than in healthy controls prior to vaccination. Mann-Whitney $U$ test was used to analyze data. ${ }^{* * * *} P \leq 0.0001$. Black circles represent data from the 2014/15 influenza season; red circles represent data from the 2015/16 influenza season.

a higher frequency of transitional B cells compared with healthy controls $(10.3 \%$ versus $5 \%$, respectively; $P$ $\leq 0.001$ ), with similar differences observed when comparing absolute counts of transitional B cells (Figure 1D). Notably, the patients recruited during early reconstitution, with low total B cell frequencies, were generally the ones with higher frequencies of transitional cells.

Lack of peripheral influenza-specific MBCs in patients previously treated with rituximab. Little information is available on the survival or potential homeostatic recovery of antigen-specific memory cells after B cell depletion in humans (14). However, it is clear that the overall MBC population remains low for long periods of time after depletion (38) (Figure 2A). Accordingly, the frequency of CD27+ MBCs of total B cells was significantly lower in patients than in healthy controls ( $4 \%$ versus $25.5 \%$, respectively; $P \leq 0.0001$ ). This was also true when comparing absolute numbers of $\mathrm{CD} 27^{+} \mathrm{MBCs}$ (Figure 2B). We next used an ELISPOT-based assay to detect stimulated antigen-specific MBCs present in both patients and healthy controls prior to vaccination (39). Importantly, we could not detect influenza-specific MBCs in almost all of the patients, with only 3 patients showing measurable numbers of influenza-specific MBCs. In contrast, these cells were detectable in all but one of the healthy controls prior to vaccination (median $0.4 \%$ ), similar to previous reports $(40,41)$ (Figure 2C). It remains unclear if the low number of MBCs in patients represents newly generated memory cells or preexisting memory cells that survived depletion. Overall, although total $\mathrm{B}$ cell numbers return to normal over time, rituximab has a profound and long-lasting effect on the MBC compartment in blood.

Potent plasmablast responses after vaccination, despite the lack of circulating influenza-specific MBCs. Previous studies have shown that the magnitude of plasmablast responses correlates with the serological outcome of vaccination and can be used as a predictor of influenza vaccination efficacy $(42,43)$. In healthy controls, these responses are primarily class-switched to the IgG isotype, peak about 7 days after vaccination, and are thought to predominantly originate from MBCs $(41,42)$. These rapid and potent anamnestic responses are transient in peripheral blood, with some plasmablasts likely taking up residence in survival niches within the bone marrow (44) or possibly other lymphoid organs. Surprisingly, we found that, despite the lack of detectable influenza-specific MBCs in peripheral blood, patients mounted potent plasmablast responses after vaccination of comparable magnitude and kinetics to those observed in healthy controls. This was shown by flow cytometric analysis, which measured plasmablast frequency (Figure 3A), as well as ELISPOT assay, which measured influenza-specific antibody-secreting cells (ASCs) (Figure 3B). The average numbers of influenza-specific ASCs per million peripheral blood mononuclear cells (PBMCs) at day 7 was similar between healthy controls and patients comparing IgG ( 820 versus 730 , respectively), IgA (330 versus 240 , respectively), and IgM ( 83 versus 22 , respectively) 
A

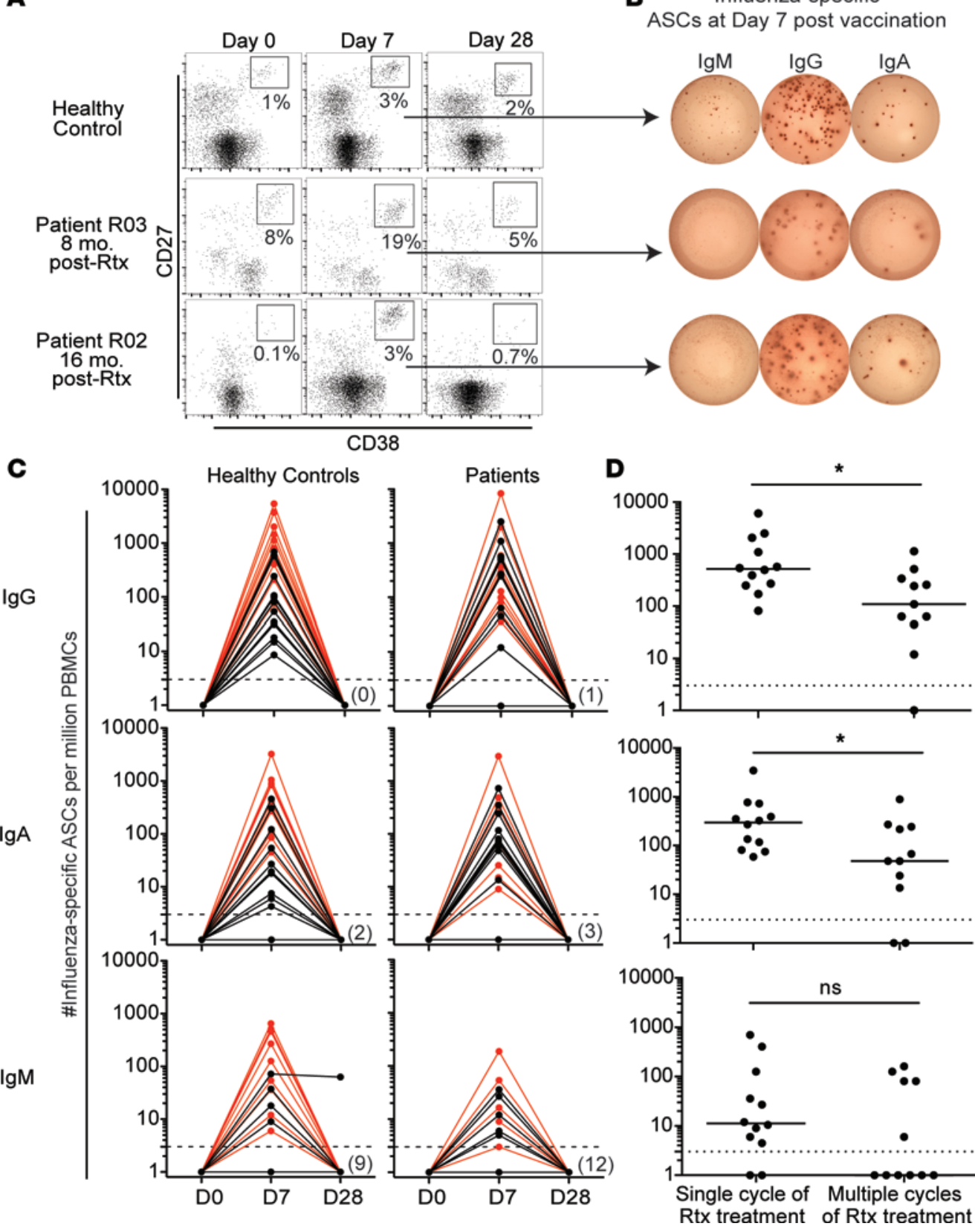

Figure 3. Robust vaccine-induced plasmablast responses likely originating from memory recall responses. (A) Representative flow cytometry plots showing transient expansion of $C D 27^{\text {hi }} C D 38^{\text {h }}$ plasmablasts, gated on CD19+ $B$ cells, induced at day 7 after vaccination. (B) Representative ELISPOT of influenza-specific plasmablasts at day 7 after vaccination. (C) Influenza-specific antibody-secreting cells (ASCs) were measured by ELISPOT at day 0,7 , and 28 after vaccination. The average number of influenza-specific ASCs per million PBMCs was plotted by lgG, IgA, and IgM isotypes. Dotted line represents the limit of detection of the ELISPOT assay. Number in parentheses represents the number of vaccinees who did not have a detectable influenza-specific plasmablast response. (D) Patients who had no history of rituximab treatment prior to enrollment had a significantly higher IgG and IgA plasmablast response to vaccination compared with patients who had received a range of 2-4 cycles of rituximab. Mann-Whitney $U$ test was used to analyze data. ${ }^{*} P$ $\leq 0.05$. Black lines represent data from the 2014/15 influenza season; red lines represent data from the 2015/16 influenza season.

isotypes. These responses were clearly dominated by class-switched antibodies in both healthy controls and patients, suggesting that these responses were of memory origin (Figure 3C). Interestingly, patients who had received multiple rituximab treatments in the past did have a significantly smaller IgG and IgA plasmablast response compared with patients who had only received a single cycle of rituximab, with one patient who had previously received 2 rounds of rituximab treatment failing to produce any detectable plasmablast response. While there was no significant difference when comparing IgM responses, we believe this is likely due to low IgM plasmablast responses in both patient cohorts (Figure 3D). This suggests that multiple rounds of rituximab may increase the level of depletion of MBCs in patients.

The magnitude of the plasmablast response does not seem to depend on the amount of time after receiving rituximab (Supplemental Figure 2A). Additionally, there was no difference in the magnitude of the plasmablast response when comparing patients who were not receiving additional immunosuppressants at time of vaccination and those who were (Supplemental Figure 2B), although this may be due to the low dose of immunosuppressants typically prescribed to AIBD patients. Finally, different types of AIBD (Supplemental Figure 2C) and relapse within 2 years of vaccination (Supplemental Figure 2D) did not have 
A

Hemagglutination Inhibition Assay

$2014 / 2015$

$2015 / 2016$

A/California/7/2009 (H1N1)

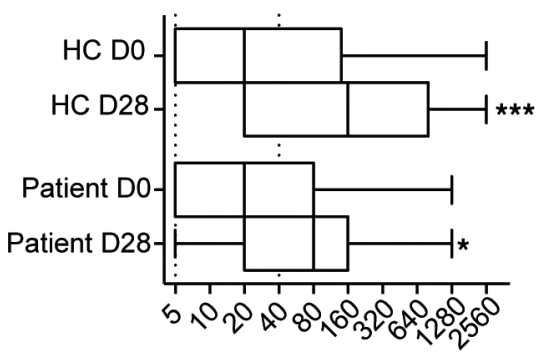

AVCalifornia/7/2009 (H1N1)

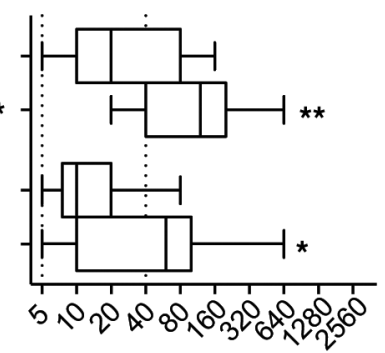

A/Switzerland/9715293/13 (H3N2)

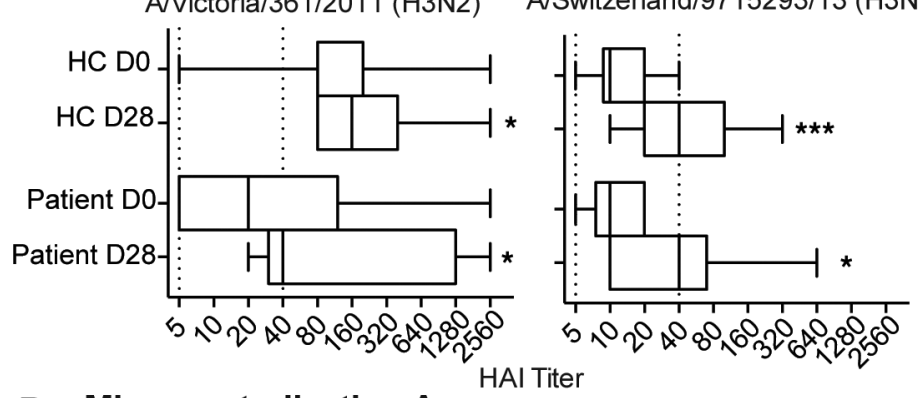

B Microneutralization Assay

2014/2015

AVCalifornia/7/2009 (H1N1)

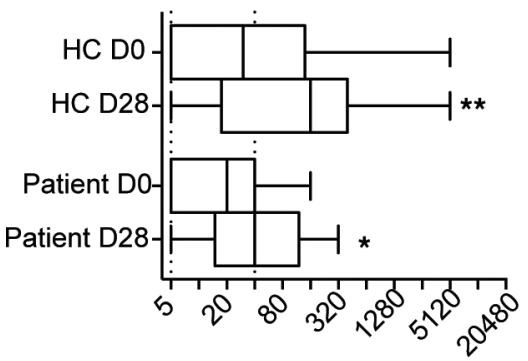

ANictoria/361/2011 (H3N2)

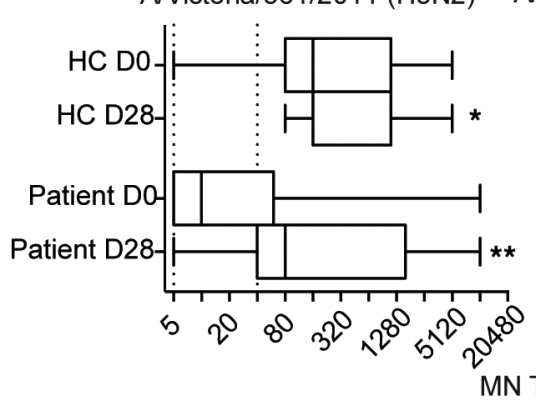

2015/2016

A/California/7/2009 (H1N1)

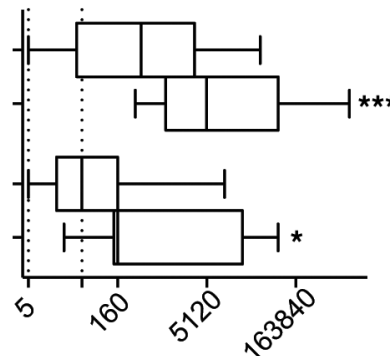

A/Switzerland/9715293/13 (H3N2)

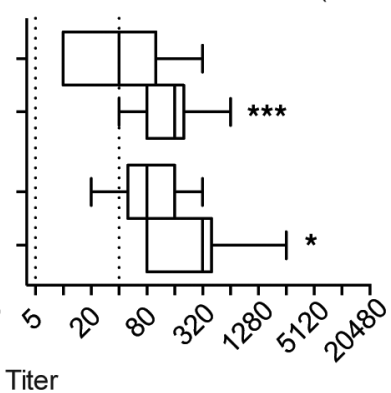

Figure 4. Comparable serological responses to vaccination in patients and healthy controls. (A) Influenza-specific serum antibody titers determined by HAI. HAI titers were plotted, comparing serum samples from day 0 to day 28 after vaccination. The dotted line represents the limit of detection of the assay at an HAI titer of 5 and seroprotection at an HAl titer of 40 . (B) Influenza-neutralizing serum antibody titers determined by microneutralization assay. Titers are graphed as a box-and-whisker plots. Horizontal lines within boxes indicate medians, while widths of boxes represent interquartile ranges. Whiskers show the highest and lowest titer values measured. The experiments were performed in duplicate and reproduced twice. One representative experiment in shown. Wilcoxon paired $t$ test was used to compare day 0 to day 28 after vaccination within cohorts. ${ }^{*} P \leq 0.05 ;{ }^{* *} P \leq 0.01 ;{ }^{* *} P \leq 0.005$.

a significant effect on the plasmablast response. From these data, we conclude that, after peripheral B cell reconstitution has begun, patients can mount a robust plasmablast response, likely originating from memory cells, despite low or undetectable numbers of MBCs in peripheral blood.

Protective serum antibody responses to vaccination in patients and healthy controls. In addition to comparable plasmablast responses, both patients and healthy controls mounted robust serological responses to the vaccine. After vaccination during the 2014/15 influenza season, hemagglutination inhibition (HAI) geometric mean titers (GMT) increased significantly $(P \leq 0.05)$ against the $\mathrm{H} 1$ strain for both patients (GMT 22-61) and healthy controls (GMT 31-145) as well as the H3 strain for patients (GMT 31-123) and healthy controls (GMT 119-215). Similar results were observed in vaccinees enrolled during the 2015/16 influenza season, with GMTs increasing in the H1 strain in patients (GMT 14-46) and healthy controls (GMT 26-98) as well as in the H3 strain in patients (GMT 12-40) and healthy controls (GMT 11-44) (Figure 4A). Additionally, the frequency of seroprotection in patients and healthy controls after vaccination was comparable, suggesting that vaccination provided similar protective immunity in both cohorts (Supplemental Figure 3A).

Microneutralization $(\mathrm{MN})$ assays showed similar results. During the 2014/15 influenza seasons, significant increases in MN GMT $(P<0.05)$ were observed against the H1 strain in patients (GMT 16-40) and healthy controls (GMT 34-125) as well as for the H3 strain in patients (GMT 29-136) and healthy controls (GMT 226-371). These increases were also observed during the 2015/16 influenza seasons against the H1 strain in patients (GMT 64-905) and healthy controls (GMT 290-6,890) as well as for the H3

strain in patients (GMT 86-260) and healthy controls (GMT 38-138) (Figure 4B). The frequency of seroprotection, as determined by $\mathrm{MN}$ assay, was comparable between patients and healthy controls, which confirmed the robustness of the vaccine responses in both cohorts (Supplemental Figure 3B). Overall, these serological analyses demonstrated that both the quantity and quality of the vaccine-induced immune response were similar for healthy controls and patients.

Rituximab does not impair the generation of antigen-specific MBCs. To evaluate if previous rituximab treatment had any effect on the generation of new MBCs, we determined the frequency of influenza-specific MBCs after vaccination. To this end, we used a 6-day polyclonal in vitro stimulation of PBMCs, causing 


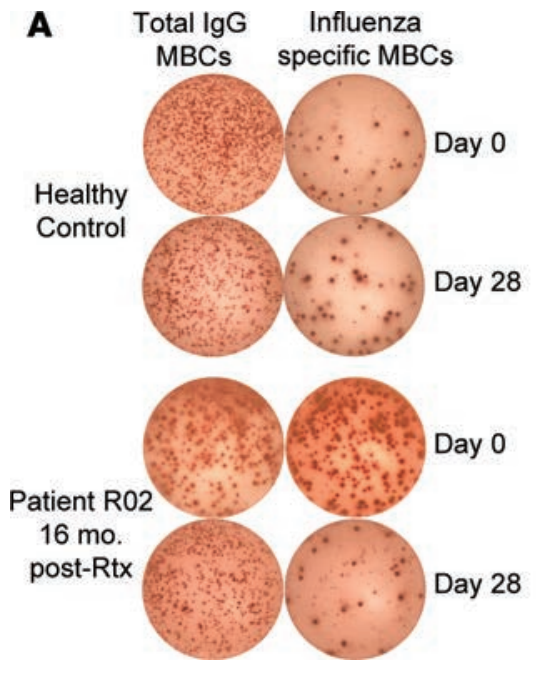

B

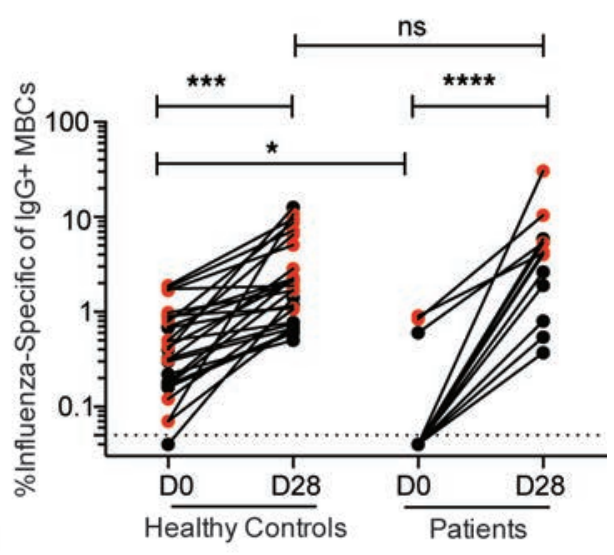

Figure 5. Previous rituximab depletion has no effect on the generation of new influenza-specific memory B cells. The frequency of influenza-vaccine specific MBCs was measured at baseline and day 28 after vaccination. (A) Representative memory B cell assay. PBMCs were stimulated with a mitogen cocktail, and the frequency of antigen-specific Ig $\mathrm{C}^{+} \mathrm{MBC}$ s of total IgG+ MBCs was determined by ELISPOT. (B) The frequency of influenza-specific lg $\mathrm{C}^{+} \mathrm{MBC}$ at day 0 and day 28 after vaccination. Only vaccinees that responded to the polyclonal stimulation were included. One-way ANOVA was used to analyze data. ${ }^{*} P \leq 0.05 ;{ }^{* *} P \leq 0.001$. Black circles represent data from the $2014 / 15$ influenza season; red circles represent data from the 2015/16 influenza season.

MBCs to differentiate into ASCs. This was followed by ELISPOT measures of both total and influenza-specific IgG-secreting cells, with responses reported as the frequency of antigen specificity per total IgG-secreting cells (39) (Figure 5A).

As mentioned previously (Figure 2C), only 3 patients had detectable influenza-specific MBCs prior to vaccination, whereas these cells were present in 23 of 24 healthy controls before vaccination. This shows that rituximab efficiently depletes peripheral antigen-specific MBCs. Strikingly, we observed a robust increase in the frequency of influenza-specific $\operatorname{IgG}^{+} \mathrm{MBC}$ from day 0 to day 28 after vaccination in both healthy controls $(0.4 \%-1.8 \%, P \leq 0.001)$ and patients $(0.0 \%-4.2 \%, P \leq 0.001)$ alike, such that a difference in the frequency at day 28 between healthy controls and patients was no longer observed (Figure 5B). This suggests that the generation of new peripheral MBCs is not impaired in these patients. Further studies on the longevity of these cells will elucidate if previous rituximab therapy has any effect on the long-term maintenance of these newly generated memory cells.

We were unable to obtain data for 10 of the patients using our standard MBC assay. Although total MBCs were detectable by flow cytometry, B cells failed to expand in vitro, presumably due to the low frequency of memory cells in the PBMC preparation (Supplemental Figure 4A). To recover some of these data points, we evaluated an alternative polyclonal stimulation method, using R848 and huIL-2 (45). This approach provided almost identical data compared with the classical method at high levels of MBCs but was much more efficient in stimulating samples with low frequencies of MBCs (Supplemental Figure 4, B and C). We were able to obtain antigen-specific MBC frequencies from an additional 6 patients using this optimized methodology. Data from these patients confirmed the findings shown in Figure 5B. All but one patient had undetectable influenza-specific MBCs prior to vaccination, with robust increases in antigen-specific memory cells from day 0 to day 28 after vaccination in all patients (median $0.0 \%-1.0 \%$ ). In contrast, all healthy controls retested with the alternative mitogen cocktail had detectable influenza-specific MBCs prior to vaccination (median $0.23 \%$ ) that expanded after vaccination (median 2.2\%) (Supplemental Figure 4D). Overall, these results show that, although rituximab successfully depletes MBCs in the periphery, patients can still develop new peripheral antigen-specific MBCs in response to an antigenic challenge.

Repertoire of vaccine-induced plasmablasts is similar between patients and healthy controls. To determine if the plasmablast responses derived from an expansion of a small number of precursor MBCs or a more diverse pool of memory cells, we analyzed the antibody repertoire breadth of plasmablasts from a subset of patients and healthy controls. Using previously published primer sets $(46,47)$, we amplified the $\mathrm{V}_{\mathrm{H}}$ gene from single cell-sorted plasmablasts using multiplex PCR and analyzed the sequences of class-switched heavy chains. Overall, we analyzed 172 sequences from 5 healthy controls and 286 sequences from 8 patients, with a range of 22-46 sequences per vaccinee (Supplemental Table 1). Comparing patients and healthy controls, we observed no difference between the average number of somatic hypermutations observed in the $\mathrm{V}_{\mathrm{H}}$ gene (21 to 20, respectively; $P=0.49$ ). The high level of mutation further confirms the memory origin of the plasmablast response in both cohorts (Figure 6A). Additionally, 
A

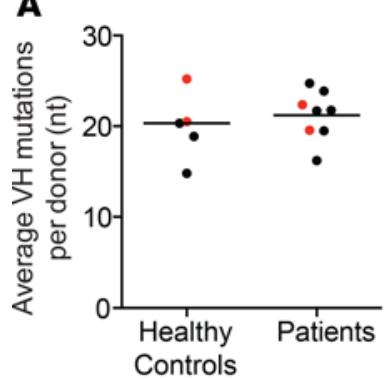

D

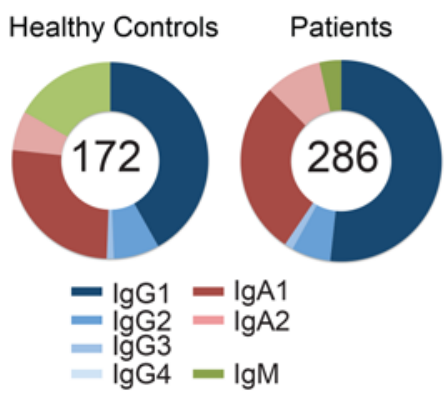

B

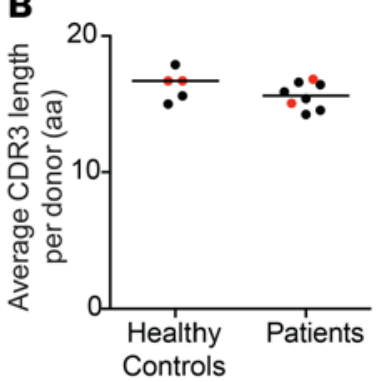

E

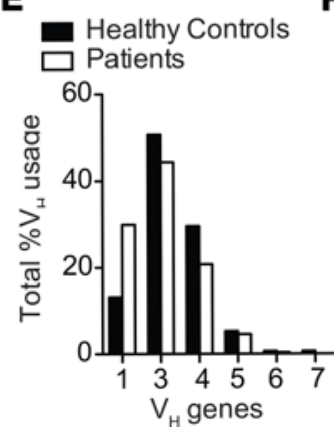

C

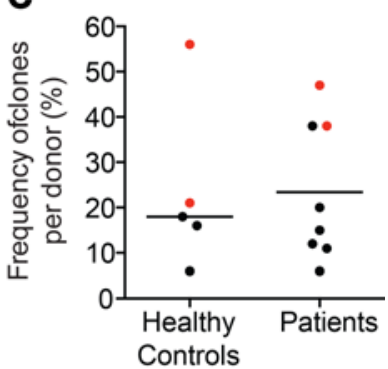

$\mathbf{F}$

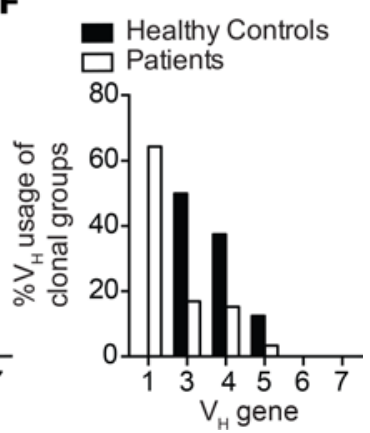

Figure 6. Vaccine-induced plasmablasts display comparable repertoire breadth in patients and healthy controls. Variable genes from plasmablasts induced by the seasonal influenza vaccine were amplified by single-cell PCR and analyzed for (A) number of somatic mutations, (B) CDR3 length, and (C) clonality (shared identical VH gene, JH gene, and CDR3 junction) of classswitched sequences. Each dot represents one individual donor, averaged from 22-46 sequences. (D) Frequency of isotypes in patients and healthy controls. A total of 172 sequences were analyzed from 5 healthy controls, and 286 sequences were analyzed from 8 patients. (E) Overall VH gene usage of class-switched sequences, reported as a frequency of total sequences analyzed from each cohort. (F) VH gene usage of antibodies in clonal groups reported as a frequency of total number of antibodies involved in clonal groups identified. Black circles represent data from the 2014/15 influenza season; red circles represent data from the 2015/16 influenza season.

there was no difference between patients and healthy controls when measuring the average CDR3 length (16.4 aa and 15.6 aa, respectively; $P=0.22$ ) (Figure $6 \mathrm{~B}$ ) as well as the average frequency of clonal expansions (23.4\% and $22.6 \%$, respectively; $P=0.93$ ) (Figure $6 \mathrm{C}$ ). Neither the number of unique clonal families nor the number of variants per family were significantly different between patients and healthy controls, further confirming that there was no difference in the repertoire breadth of plasmablast responses between the two cohorts (data not shown). Since the plasmablast responses were not clonally restricted in patients but as diverse as those observed in healthy controls, we conclude that this likely means that the plasmablast response originated from a diverse pool of MBCs rather than from the small number of MBCs present in peripheral blood. This suggests that tissue-resident MBCs are able to survive rituximab-mediated depletion, likely in lymphoid organs (14), despite circulating memory cells being fully depleted.

Because pemphigus has been associated with a bias toward serum IgG1 and IgG4 (48), we characterized the isotype usage in the plasmablast responses. Overall, there was a similar distribution of isotypes comparing patients and healthy controls, with no IgG4 detected in either cohort (Figure 6D). Although the distribution of $\mathrm{V}_{\mathrm{H}}$ gene was similar between healthy controls and patients (Figure 6E), patients had a bias of $\mathrm{V}_{\mathrm{H}} 1$ gene usage in clonally expanded populations that was not observed in healthy controls (Figure $6 \mathrm{~F}$ ). Overall, sequence analysis shows that plasmablasts likely originate from a diverse pool of memory cells in both patients and healthy controls and suggests that there is little effect of rituximab or autoimmune disease on the quality and repertoire of the plasmablast response.

\section{Discussion}

In this study, we observed robust influenza vaccine responses in rituximab-treated AIBD patients, comparable to those mounted in healthy controls. To our knowledge, this is the first study to assess these responses in rituximab-treated AIBD patients. AIBD patients are typically given rituximab with a tapering course of low-dose systemic corticosteroids, which gives us an opportunity to study vaccine responses in patients that received B cell ablative therapy but are not taking additional immunosuppressants at the time of vaccination. This is different from other rituximab-treated patient populations, who are typically prescribed immunosuppressants or chemotherapy both during and after rituximab treatment. Additionally, underlying autoimmune disease may differentially effect vaccine responses after rituximab treatment. For example, AIBD has well-defined antigenic targets confined to the skin, which is different from the complexity of other systemic B cell-mediated diseases, such as RA (49) or lymphoma (50). These differences may explain the robust vaccine responses observed in this study, which differ from previous reports that have suggested that rituximab impairs influenza vaccine responses (30-34). 
The majority of patients enrolled in this study had a repopulated B cell compartment, with normal total B cell numbers in peripheral blood. However, patients had significantly fewer peripheral MBCs than healthy controls. Moreover, while almost all healthy controls had influenza-specific MBCs prior to vaccination, these cells were undetectable in all but 3 of the rituximab-treated patients. Because these 3 patients had not been vaccinated within the last 3 years, it is likely that these cells are a result of a recent infection or MBCs that survived depletion. Surprisingly, despite the impaired memory compartment, patients retained their ability to mount a recall response to the influenza vaccine. This was evident by potent plasmablast responses of comparable magnitude, kinetics, and immunoglobulin isotype to those observed in healthy controls as well as normal expansion of influenza-specific MBCs after vaccination. Additionally, patients mounted serological responses similar in magnitude and quality to healthy controls. Importantly, the patients that had received multiple cycles of rituximab displayed reduced responses (Figure 3), suggesting that multiple cycles might provide a more complete depletion of MBCs in tissues. One patient failed to mount a plasmablast response altogether, despite having a normal number of peripheral B cells at 16 months after rituximab and not taking any additional immunosuppressants. Interestingly, this patient had previously received 2 previous rounds of rituximab. This finding is supported by a previous study in which patients who had previously received maintenance rituximab monotherapy to treat neuromyelitis optica appeared to have blunted vaccine responses (32), suggesting that repeat dosages or rituximab may be associated with poor influenza vaccine efficacy. It is possible that the repeat treatments caused a more complete depletion, specifically in lymphoid tissues, which could explain the lack of plasmablast response. Additional studies of the effect of multiple depletions on tissue-resident MBCs and subsequent vaccine responses may be warranted in order to better optimize rituximab dosages and schedules for patients and to further determine the optimal therapy to treat autoimmune disorders.

Repertoire analysis of plasmablast responses in both patients and healthy controls revealed highly mutated immunoglobulin sequences, further confirming the memory origins of these responses. Additionally, patients and healthy controls had similar frequencies of clonal expansions in their plasmablast responses, which suggests that these cells originated from a diverse pool of memory cells, rather than from a rapid expansion of the small number of peripheral blood MBCs. Based on a previous observation in splenectomized immune thrombocytopenia patients that revealed the presence of persisting antigen-specific MBCs in the spleen $(14,51)$, we believe it is likely that this diverse pool of cells survives in tissues. Additionally, a recent study showed that tissue-resident MBCs in a mouse model are enriched for broadly neutralizing influenza-specific antibodies compared with those in circulation (52). If influenza vaccine responses in patients are originating from a diverse pool of tissue-resident MBCs, this mouse study could potentially explain how patients are able to mount a protective response to vaccination despite the low number of MBCs.

Despite previous observations that both autoimmune disease pathogenesis $(48,53)$ and rituximab therapy (54) may effect the overall immunoglobulin diversity, we observed no unique mutation frequency, CDR3 length, or isotype frequency in the plasmablast population of AIBD patients compared with healthy controls. Although there was no difference in overall $\mathrm{V}_{\mathrm{H}}$ gene usage between patients and healthy controls, patients did have a bias in $\mathrm{V}_{\mathrm{H}} 1$ gene usage in clonal expansions. This may be an interesting finding considering recent reports that have observed a bias of $\mathrm{V}_{\mathrm{H}} 1-69$ gene usage in broadly neutralizing antibodies (55) as well as a potential role for $\mathrm{V}_{\mathrm{H}} 1-46$ gene usage in pemphigus pathogenesis (56). Future experiments with monoclonal antibodies selected from these clonal expansions may reveal subtle differences in the quality of antibody responses comparing healthy controls and rituximab-treated AIBD patients.

Previous observations have suggested that some B cells survive depletion after rituximab therapy within secondary lymphoid organs $(38,57-62)$. However, there are very few studies of this in humans, and it is often difficult to interpret the data from these studies due to low sample numbers and patient-to-patient variability. To our knowledge, there is only one report of human antigen-specific MBCs surviving in the spleen after rituximab depletion (14). Several mechanisms underlying the persistence of B cells in tissues have been proposed, including resistance of activated B cells to rituximab-mediated depletion $(61,63)$, effect of Fc glycosylation on rituximab and its resulting interaction with $\mathrm{Fc}_{\mathrm{C}}$ receptors on accessory cells (64), the inflammatory or cytokine milieu allowing for B cells to survive $(65,66)$, or possibly the inability of rituximab to fully access tissues to deplete B cells (67). It is also possible that CD20 expression is downregulated in $\mathrm{B}$ cells residing in tissues. Although a large number of studies have shown that MBCs in the spleen (65), bone marrow (62), human blood, and lymph node (68) general coexpress CD19 and CD20 at high levels, we cannot out rule the possibility that a small number of MBCs downregulate CD20, similar 
to plasma cells (11). It is also possible that a combination of several of the proposed mechanisms work in concert to effect $\mathrm{B}$ cell depletion in tissues. Consistent with these findings, our data suggest that tissue-resident MBCs survive rituximab-mediated depletion and maintain the ability to initiate recall responses upon antigenic challenge. While this may be beneficial for vaccine responses, it is possible that surviving MBCs may also paradoxically act as a source for pathogenic responses, causing relapse in autoimmune disease. In fact, previous reports have detected persisting clones of autoimmune MBCs after rituximab treatment, both in pemphigus and anti-MAG neuropathy $(54,69)$. Given that almost all diseases currently treated with rituximab depletion have a tendency to relapse, including other autoimmune diseases, such as RA and systemic lupus erythematosus, as well as B cell lymphomas, it is critically important to understand how tissue-resident MBCs survive depletion and their role in both protective and pathogenic recall responses. These findings indicate that future analyses of immunological memory and the development of new generations of antibody-based B cell depletion strategies should focus on ways to optimize the depletion of $\mathrm{MBC}$ in tissues as a way to minimize relapsing disease. These new strategies may include improving the current rituximab therapy by modulating its $\mathrm{Fc}$ domain to improve its effector function (70) or the use of CD19 as a target for depletion of B cells (71).

Overall, these data show that, although the patients lacked preexisting antigen-specific MBCs in circulation prior to vaccination, they were uniformly able to respond to vaccination, with a response of similar magnitude, quality, and repertoire breadth as that found in healthy controls. This suggests that a substantial pool of noncirculating MBCs remains present in lymphoid tissues after depletion and can serve to seed these responses. These findings have important implications for vaccination strategies for AIBD patients and other rituximab-treated patients and contribute to our understanding of MBC homeostasis and survival.

\section{Methods}

Study design. Subjects received a single dose of commercially available trivalent, inactivated influenza vaccine administered intramuscularly. For the 2014/15 influenza season, vaccine (Flucelvax, Novartis) contained the following influenza strains: A/Brisbane/10/2010 (H1N1, A/California/7/2009-like virus), NYMC X-223A (H3N2, A/Victoria/361/2011-like virus), and B/Massachusetts/2/2012. During the 2015/16 influenza season, vaccine (Fluvirin, Novartis) contained the following influenza strains: A/Christchurch/16/2010, NIB74 (H1N1, A/California/7/2009 pdm09-like virus), NIB-88 (H3N2, A/Switzerland/9715293/2013-like virus), and B/Phuket/3073/2013. Blood sample were collected in sodium citrate CPT tubes (BD Vacutainer) at baseline, day 7 ( \pm 1 day), and day 28 ( \pm 5 days) after vaccination. Plasma samples were collected and stored at $-80^{\circ} \mathrm{C}$. PBMCs were isolated and washed with PBS $/ 2 \% \mathrm{FBS}$. Cells were used fresh or were frozen in FBS/10\% dimethyl sulfoxide. Frozen PBMCs were stored in liquid nitrogen until assayed.

Flow cytometry analysis and single-cell sorting. Immunophenotyping of circulating B cell subpopulations was performed on fresh whole blood stained with the following mAbs, appropriately titrated, followed by lysis of erythrocytes (BD FACS lysis solution): CD19-FITC (BD, clone HIB19), CD24-PerCPCy5.5 (Biolegend, clone ML5), CD3-PacificBlue (BD, clone SP34-2), CD38-PE (BD, clone HIT2), CD20-PECy7 (BD, L27), IgD-PECy7 (Biolegend, clone IA6-2), and CD27-APC (eBiosciences, clone O323). 100,000 events were acquired on a BD FACSCantoII flow cytometer and analyzed using FlowJo software. Plasmablasts were single-cell sorted into 96-well PCR plates containing hypotonic catch buffer with RNase inhibitor (Promega) using a FACSAriaII and were frozen immediately on dry ice, as previously described (41). Additional plasmablasts were bulk sorted into RPMI supplemented with penicillin/streptomycin, L-glutamine, and $10 \%$ FBS (R10).

Total B cell counts. Total lymphocyte counts for patients enrolled in 2014/15 were determined by complete blood counts routinely performed during patient visits to the clinic. Total lymphocyte counts for 2014/15 healthy controls were estimated using the average number of peripheral lymphocytes, as reported by the Mayo Clinic (72). TruCount tubes (BD) were used during the 2015/16 influenza season to count B cells. $50 \mu \mathrm{l}$ of blood was added to TruCount tubes and incubated for 20 minutes with CD45PE (Biolegend, clone HI30) and CD3-Pacific Blue (BD). $450 \mu 1$ of FACs lysis solution (BD) was added, and the mixture was incubated for 20 minutes before the sample was run on a BD FACSCantoII and analyzed using FlowJo software.

ELISPOT assay. ELISPOT was performed to enumerate influenza-specific plasmablasts present in both PBMC samples and bulk-sorted plasmablasts. 96-well ELISPOT assay filter plates (Millipore) were coated overnight with either influenza vaccine (1:20, Novartis) or polyvalent goat anti-human Ig $(10 \mu \mathrm{g} / \mathrm{ml}$, 
Jackson ImmunoResearch) in PBS. Plates were washed and blocked by incubation with R10 at $37^{\circ} \mathrm{C}$ for 2 hours. Freshly isolated PBMCs were added to the plates in a dilution series starting at $5 \times 10^{5}$ cells and incubated overnight at $37^{\circ} \mathrm{C}$. Plates were washed with PBS, followed by PBS $/ 0.05 \%$ Tween and then incubated with biotinylated anti-human IgG, IgA, or IgM antibody (Invitrogen) at room temperature for 90 minutes. After washing, plates were incubated with avidin D-horseradish peroxidase conjugate (Vector laboratories) and developed using 3-amino-9-ethyl-carbazole substrate (Sigma-Aldrich). Plates were scanned and analyzed using an automated ELISPOT counter (CTL, Cellular Technologies).

$M B C$ assay. Antigen-specific MBCs were detected essentially as previously described (39). In brief, PBMCs were cultured at $1 \times 10^{6}$ cells per $\mathrm{ml}$ of R10 supplemented with $50 \mu \mathrm{M} \beta$-mercaptoethanol (Sigma-Aldrich) and polyclonally stimulated with pokeweed mitogen extract ( $1 \mu \mathrm{g} / \mathrm{ml}$, Sigma-Aldrich), phosphothiolated CpG ODN-2006 (6 $\mu \mathrm{g} / \mathrm{ml}$, Invivogen), and Staphylococcus aureus Cowan (1:10,000, Sigma-Aldrich) for 6 days. After in vitro stimulation, total and influenza-specific IgG-secreting cells were quantified by ELISPOT assay, as described above.

HAI assay. HAI assay was performed as per World Health Organization criteria (73). Briefly, plasma samples were treated with receptor-destroying enzyme (Denka Seiken) and heat inactivated. Samples were serially diluted in PBS in a 96-well v-bottom plate with 8 HAU of live, egg-grown virus added to the well. Viruses were provided by A. Ellebedy (Emory University, Atlanta, Georgia, USA), A. Lowen (Emory University), and S. Gangappa (CDC, Atlanta, Georgia, USA). After a 30-minute incubation at room temperature, 501 of $0.5 \%$ turkey RBCs (Lampire) suspended in PBS was added to each well. After a 30-minute incubation at room temperature, HAI titers were determined based on the final dilution for which agglutination inhibition was observed.

MN assay. MN assays were performed as previously described by the World Health Organization (74). Briefly, plasma was heat-inactivated and serially diluted in DMEM with 10\% BSA, 2\% HEPES, and penicillin/streptomycin. Virus diluted at $\times 100$ TCID50 per $50 \mu 1$, as determined by the Reed-Muench method, was added, and the mixture was incubated at $37^{\circ} \mathrm{C}$ for 1 hour. MDCK cells (ATCC), diluted at $1.5 \times 10^{5}$ cells $/ \mathrm{ml}$, were added to the plate and incubated for another 20 hours at $37^{\circ} \mathrm{C}$. The plate was washed with PBS and fixed with cold $80 \%$ acetone in PBS. Plates were washed with PBS/Tween20 and incubated with anti-influenza NP monoclonal antibody (Millipore) and diluted 1:1,000 in 5\% milk for 1 hour at room temperature. After washing, HRP-conjugated goat anti-mouse IgG (Kirkegaard and Perry Laboratories) diluted at 1:2,000 in milk was added and incubated for another hour at room temperature. After the wash, plates were developed for 8 to 12 minutes using OPD tablets (Sigma-Aldrich P8787) diluted in citrate buffer (Sigma-Aldrich P4560). Absorbance readings were measured at OD490 on a microplate reader. MN titers were reported as the serum dilution corresponding to $50 \%$ neutralization.

Repertoire analysis. Identification of antibody variable region genes was performed essentially as previously described $(41,46)$. In brief, single-cell cDNA was synthesized from sorted plasmablasts using random hexamers (Sensiscript, Qiagen). Ig heavy chain rearrangements were then amplified by nested PCR (HotStarTaq Plus Master Mix, Qiagen) using primer cocktails specific for all V gene families and constant domains at a concentration of $200 \mathrm{nM}$ per primer (46). Sense primers used in the second round of nested PCR were modified by fusing the $5^{\prime}$ end of each primer to the M13R sequence (5'-AACAGCTATGACCATG-3') to facilitate subsequent sequencing. V, D, and J genes were identified and analyzed using the ImMunoGeneTics (IMGT) database as a reference. Somatic hypermutation levels in the $\mathrm{V}_{\mathrm{H}}$ genes represent the number of mutations in the amplicon (FR1 through CDR3) relative to the closest germline sequence matched in the IMGT database. Clonal expansions were determined by sequence alignments of rearrangements with matching $\mathrm{V}$ and $\mathrm{J}$ gene usage, where cells with identical junctional diversity were grouped as part of the same clonal expansion.

Statistics. Data were collected and graphed using GraphPad Prism software. A Mann-Whitney $U$ test, Wilcoxon matched-pairs test, or 1-way ANOVA Kruskal-Wallis test was used to determine statistical significance where appropriate. Seroprotection was analyzed using a Fisher exact test. $P$ values of less than 0.05 (2 sided) were considered statistically significant.

Study approval. Patient volunteers were informed of the study during routine clinical visits at the Department of Dermatology and enrolled based on interest in receiving seasonal influenza vaccination during the $2014 / 15$ or 2015/16 influenza seasons. Patient inclusion criteria included age $\geq 18$ years old and treatment with rituximab for AIBD within the last 24 months. Demographically matched healthy volunteers were recruited from staff and students receiving the influenza vaccination at Emory University. Written informed 
consent was obtained from all participants. Exclusion criteria included prior vaccination within the current season as well as any condition that, in the opinion of the investigator, would place the subject at risk of injury from participation. This study was approved by the Emory Institutional Review Board (IRB 00069980).

Note added in proof. On March 23, 2017, rituximab treatment for AIBD was formally approved by the FDA.

\section{Author contributions}

AC carried out experiments, analyzed the data, and wrote the manuscript. BB provided clinical support of the study. RK, LP, and YK contributed to sample collection and analysis, critical discussion, and editing of this manuscript. RF and JW conceived the research, oversaw the experiments and data analysis, and wrote and approved the final manuscript.

\section{Acknowledgments}

We thank R. Karaffa and S. Durham at the Emory School of Medicine flow cytometry core and A. Rae at Emory Pediatric's flow cytometry core for their technical assistance with cell-sorting experiments. We would also like to thank A. Ellebedy, A. Lowen, and S. Gangappa for providing the influenza virus strains for our experiment. We also thank the Dermatology Foundation for support of our work through a Career Development Award to RF. This study was supported by the National Institutes of Health (U19 AI057266) and Centers of Excellence of Influenza Research and Surveillance (HHSN 255200700006Z).

Address correspondence to: Jens Wrammert, Department of Pediatrics, Division of infectious Disease, Emory Vaccine Center, School of Medicine, Emory University, 1760 Haygood Drive, HSRB building E480, Atlanta, Georgia 30322, USA. Phone: 404.778.3265; Email: jwramme@emory.edu.

1. Thompson WW, et al. Influenza-associated hospitalizations in the United States. JAMA. 2004;292(11):1333-1340

2. Thompson WW, et al. Mortality associated with influenza and respiratory syncytial virus in the United States. JAMA. 2003;289(2):179-186.

3. Kunisaki KM, Janoff EN. Influenza in immunosuppressed populations: a review of infection frequency, morbidity, mortality, and vaccine responses. Lancet Infect Dis. 2009;9(8):493-504

4. Govaert TM, Thijs CT, Masurel N, Sprenger MJ, Dinant GJ, Knottnerus JA. The efficacy of influenza vaccination in elderly individuals. A randomized double-blind placebo-controlled trial. JAMA. 1994;272(21):1661-1665.

5. Potter CW, Oxford JS. Determinants of immunity to influenza infection in man. Br Med Bull. 1979;35(1):69-75.

6. Du J, et al. Structural basis for recognition of CD20 by therapeutic antibody rituximab. J Biol Chem. 2007;282(20):15073-15080.

7. U.S. Food and Drug Administration. Rituximab (marketed as Rituxan) Information. http://www.fda.gov/Drugs/DrugSafety/ PostmarketDrugSafetyInformationforPatientsandProviders/ucm109106.htm. Updated July 23, 2015. Accessed May 17, 2017.

8. Gürcan HM, Keskin DB, Stern JN, Nitzberg MA, Shekhani H, Ahmed AR. A review of the current use of rituximab in autoimmune diseases. Int Immunopharmacol. 2009;9(1):10-25.

9. Nagel A, Hertl M, Eming R. B-cell-directed therapy for inflammatory skin diseases. J Invest Dermatol. 2009;129(2):289-301.

10. Hoyer BF, Manz RA, Radbruch A, Hiepe F. Long-lived plasma cells and their contribution to autoimmunity. Ann N Y Acad Sci. 2005;1050:124-133.

11. Halliley JL, et al. Long-lived plasma cells are contained within the CD19(-)CD38(hi)CD138(+) subset in human bone marrow. Immunity. 2015;43(1):132-145.

12. Breedveld F, et al. Rituximab pharmacokinetics in patients with rheumatoid arthritis: B-cell levels do not correlate with clinical response. J Clin Pharmacol. 2007;47(9):1119-1128.

13. Sutter JA, et al. A longitudinal analysis of SLE patients treated with rituximab (anti-CD20): factors associated with B lymphocyte recovery. Clin Immunol. 2008;126(3):282-290.

14. Mamani-Matsuda M, et al. The human spleen is a major reservoir for long-lived vaccinia virus-specific memory B cells. Blood. 2008;111(9):4653-4659.

15. King C, Ilic A, Koelsch K, Sarvetnick N. Homeostatic expansion of T cells during immune insufficiency generates autoimmunity. Cell. 2004;117(2):265-277.

16. Baum S, Sakka N, Artsi O, Trau H, Barzilai A. Diagnosis and classification of autoimmune blistering diseases. Autoimmun Rev. 2014;13(4-5):482-489.

17. Bhol K, Natarajan K, Nagarwalla N, Mohimen A, Aoki V, Ahmed AR. Correlation of peptide specificity and IgG subclass with pathogenic and nonpathogenic autoantibodies in pemphigus vulgaris: a model for autoimmunity. Proc Natl Acad Sci USA. 1995;92(11):5239-5243.

18. Cirillo N, Al-Jandan BA. Desmosomal adhesion and pemphigus vulgaris: the first half of the story. Cell Commun Adhes. 2013;20(1-2):1-10

19. Amagai M, Koch PJ, Nishikawa T, Stanley JR. Pemphigus vulgaris antigen (desmoglein 3) is localized in the lower epidermis, the site of blister formation in patients. J Invest Dermatol. 1996;106(2):351-355.

20. Amagai M, Stanley JR. Desmoglein as a target in skin disease and beyond. J Invest Dermatol. 2012;132(3 Pt 2):776-784.

21. Giudice GJ, Emery DJ, Diaz LA. Cloning and primary structural analysis of the bullous pemphigoid autoantigen BP180. J Invest 
Dermatol. 1992;99(3):243-250.

22. Stanley JR, Hawley-Nelson P, Yuspa SH, Shevach EM, Katz SI. Characterization of bullous pemphigoid antigen: a unique basement membrane protein of stratified squamous epithelia. Cell. 1981;24(3):897-903.

23. Almugairen $\mathrm{N}$, et al. Assessment of the rate of long-term complete remission off therapy in patients with pemphigus treated with different regimens including medium- and high-dose corticosteroids. J Am Acad Dermatol. 2013;69(4):583-588.

24. Cianchini G, Lupi F, Masini C, Corona R, Puddu P, De Pità O. Therapy with rituximab for autoimmune pemphigus: results from a single-center observational study on 42 cases with long-term follow-up. J Am Acad Dermatol. 2012;67(4):617-622.

25. Feldman RJ, Ahmed AR. Relevance of rituximab therapy in pemphigus vulgaris: analysis of current data and the immunologic basis for its observed responses. Expert Rev Clin Immunol. 2011;7(4):529-541.

26. Schmidt E, Goebeler M, Zillikens D. Rituximab in severe pemphigus. Ann N Y Acad Sci. 2009;1173:683-691.

27. Joly P, et al. A single cycle of rituximab for the treatment of severe pemphigus. N Engl J Med. 2007;357(6):545-552.

28. Ahmed AR, Spigelman Z, Cavacini LA, Posner MR. Treatment of pemphigus vulgaris with rituximab and intravenous immune globulin. N Engl J Med. 2006;355(17):1772-1779.

29. Cianchini G, Corona R, Frezzolini A, Ruffelli M, Didona B, Puddu P. Treatment of severe pemphigus with rituximab: report of 12 cases and a review of the literature. Arch Dermatol. 2007;143(8):1033-1038.

30. Bedognetti D, et al. Impaired response to influenza vaccine associated with persistent memory B cell depletion in non-Hodgkin's lymphoma patients treated with rituximab-containing regimens. J Immunol. 2011;186(10):6044-6055.

31. Eisenberg RA, et al. Rituximab-treated patients have a poor response to influenza vaccination. J Clin Immunol. 2013;33(2):388-396

32. Kim W, et al. Reduced antibody formation after influenza vaccination in patients with neuromyelitis optica spectrum disorder treated with rituximab. Eur J Neurol. 2013;20(6):975-980.

33. Oren S, et al. Vaccination against influenza in patients with rheumatoid arthritis: the effect of rituximab on the humoral response. Ann Rheum Dis. 2008;67(7):937-941.

34. van Assen $\mathrm{S}$, et al. Humoral responses after influenza vaccination are severely reduced in patients with rheumatoid arthritis treated with rituximab. Arthritis Rheum. 2010;62(1):75-81.

35. Colliou N, et al. Long-term remissions of severe pemphigus after rituximab therapy are associated with prolonged failure of desmoglein B cell response. Sci Transl Med. 2013;5(175):175ra30.

36. Mouquet $\mathrm{H}$, et al. B-cell depletion immunotherapy in pemphigus: effects on cellular and humoral immune responses. $J$ Invest Dermatol. 2008;128(12):2859-2869.

37. Palanichamy A, et al. Novel human transitional B cell populations revealed by B cell depletion therapy. J Immunol. 2009;182(10):5982-5993.

38. Anolik JH, et al. Delayed memory B cell recovery in peripheral blood and lymphoid tissue in systemic lupus erythematosus after B cell depletion therapy. Arthritis Rheum. 2007;56(9):3044-3056.

39. Crotty S, Aubert RD, Glidewell J, Ahmed R. Tracking human antigen-specific memory B cells: a sensitive and generalized ELISPOT system. J Immunol Methods. 2004;286(1-2):111-122.

40. Sasaki S, et al. Comparison of the influenza virus-specific effector and memory B-cell responses to immunization of children and adults with live attenuated or inactivated influenza virus vaccines. $J$ Virol. 2007;81(1):215-228

41. Wrammert J, et al. Rapid cloning of high-affinity human monoclonal antibodies against influenza virus. Nature. 2008;453(7195):667-671.

42. Li GM, et al. Pandemic H1N1 influenza vaccine induces a recall response in humans that favors broadly cross-reactive memory B cells. Proc Natl Acad Sci USA. 2012;109(23):9047-9052.

43. Nakaya HI, et al. Systems biology of vaccination for seasonal influenza in humans. Nat Immunol. 2011;12(8):786-795.

44. Nutt SL, Hodgkin PD, Tarlinton DM, Corcoran LM. The generation of antibody-secreting plasma cells. Nat Rev Immunol. 2015;15(3):160-171.

45. Pinna D, Corti D, Jarrossay D, Sallusto F, Lanzavecchia A. Clonal dissection of the human memory B-cell repertoire following infection and vaccination. Eur J Immunol. 2009;39(5):1260-1270.

46. Smith K, et al. Rapid generation of fully human monoclonal antibodies specific to a vaccinating antigen. Nat Protoc. 2009;4(3):372-384.

47. Tiller T, Meffre E, Yurasov S, Tsuiji M, Nussenzweig MC, Wardemann H. Efficient generation of monoclonal antibodies from single human B cells by single cell RT-PCR and expression vector cloning. J Immunol Methods. 2008;329(1-2):112-124

48. Futei Y, Amagai M, Ishii K, Kuroda-Kinoshita K, Ohya K, Nishikawa T. Predominant IgG4 subclass in autoantibodies of pemphigus vulgaris and foliaceus. J Dermatol Sci. 2001;26(1):55-61.

49. Robert-Pachot M, et al. A new target for autoantibodies in patients with rheumatoid arthritis. Ann N Y Acad Sci. 2007;1108:382-391.

50. Shankland KR, Armitage JO, Hancock BW. Non-Hodgkin lymphoma. Lancet. 2012;380(9844):848-857.

51. Giesecke C, et al. Tissue distribution and dependence of responsiveness of human antigen-specific memory B cells. $J$ Immunol. 2014;192(7):3091-3100

52. Adachi Y, et al. Distinct germinal center selection at local sites shapes memory B cell response to viral escape. J Exp Med. 2015;212(10):1709-1723.

53. Sanz I. Multiple mechanisms participate in the generation of diversity of human H chain CDR3 regions. J Immunol. 1991;147(5):1720-1729

54. Maurer MA, et al. Rituximab induces sustained reduction of pathogenic B cells in patients with peripheral nervous system autoimmunity. J Clin Invest. 2012;122(4):1393-1402.

55. Wrammert J, et al. Broadly cross-reactive antibodies dominate the human B cell response against 2009 pandemic H1N1 influenza virus infection. J Exp Med. 2011;208(1):181-193.

56. Cho MJ, et al. Determinants of VH1-46 cross-reactivity to pemphigus vulgaris autoantigen desmoglein 3 and rotavirus antigen VP6. J Immunol. 2016;197(4):1065-1073

57. Doorenspleet ME, et al. Rheumatoid arthritis synovial tissue harbours dominant B-cell and plasma-cell clones associated with autoreactivity. Ann Rheum Dis. 2014;73(4):756-762. 
58. Schröder C, Azimzadeh AM, Wu G, Price JO, Atkinson JB, Pierson RN. Anti-CD20 treatment depletes B-cells in blood and lymphatic tissue of cynomolgus monkeys. Transpl Immunol. 2003;12(1):19-28.

59. Audia S, et al. Immunologic effects of rituximab on the human spleen in immune thrombocytopenia. Blood. 2011;118(16):4394-4400.

60. Leandro MJ, Cooper N, Cambridge G, Ehrenstein MR, Edwards JC. Bone marrow B-lineage cells in patients with rheumatoid arthritis following rituximab therapy. Rheumatology (Oxford). 2007;46(1):29-36.

61. Nakou M, et al. Rituximab therapy reduces activated B cells in both the peripheral blood and bone marrow of patients with rheumatoid arthritis: depletion of memory B cells correlates with clinical response. Arthritis Res Ther. 2009;11(4):R131.

62. Teng YK, et al. Immunohistochemical analysis as a means to predict responsiveness to rituximab treatment. Arthritis Rheum 2007;56(12):3909-3918

63. Adlowitz DG, et al. Expansion of activated peripheral blood memory B cells in rheumatoid arthritis, impact of B cell depletion therapy, and biomarkers of response. PLoS ONE. 2015;10(6):e0128269.

64. Anolik JH, et al. The relationship of FcgammaRIIIa genotype to degree of B cell depletion by rituximab in the treatment of systemic lupus erythematosus. Arthritis Rheum. 2003;48(2):455-459.

65. Mahévas M, et al. B cell depletion in immune thrombocytopenia reveals splenic long-lived plasma cells. J Clin Invest. 2013;123(1):432-442.

66. Wieland A, et al. Antibody effector functions mediated by Fc $\gamma$-receptors are compromised during persistent viral infection. Immunity. 2015;42(2):367-378.

67. Vos K, Thurlings RM, Wijbrandts CA, van Schaardenburg D, Gerlag DM, Tak PP. Early effects of rituximab on the synovial cell infiltrate in patients with rheumatoid arthritis. Arthritis Rheum. 2007;56(3):772-778.

68. Colovai AI, et al. Flow cytometric analysis of normal and reactive spleen. Mod Pathol. 2004;17(8):918-927.

69. Hammers CM, et al. Persistence of anti-desmoglein $3 \mathrm{IgG}(+)$ B-cell clones in pemphigus patients over years. J Invest Dermatol. 2015;135(3):742-749.

70. Teeling JL, et al. The biological activity of human CD20 monoclonal antibodies is linked to unique epitopes on CD20. J Immunol. 2006;177(1):362-371.

71. Hammer O. CD19 as an attractive target for antibody-based therapy. MAbs. 2012;4(5):571-577.

72. CBC with Differential, Blood. Mayo Medical Laboratories. http://www.mayomedicallaboratories.com/test-catalog/ Clinical+and+Interpretive/9109. Accessed May 17, 2017

73. WHO Manual on Animal Influenza Diagnosis and Surveillance. World Health Organization. http://www.who.int/csr/resources/ publications/influenza/whocdscsrncs20025rev.pdf. Accessed May 17, 2017.

74. Serological diagnosis of influenza by microneutralization assay. WorldHealth Organization. http://www.who.int/influenza/ gisrs_laboratory/2010_12_06_serological_diagnosis_of_influenza_by_microneutralization_assay.pdf. Accessed May 17, 2017. 